\title{
SCENT INTEGRATED TOOLBOX FOR MONITORING FLOOD PHENOMENA
}

\author{
MARIA KROMMYDA ${ }^{1}$, SPYROS BOLIERAKIS ${ }^{1}$, YANNIS KOPSINIS ${ }^{1}$, CHRYSOVALANTIS TSIAKOS ${ }^{1}$, \\ ATHANASIA TSERTOU ${ }^{1}$, ANGELOS AMDITIS ${ }^{1}$, ANDREJA JONOSKI ${ }^{2}$, IOANA POPESCU ${ }^{2}$, \\ DANIELE MIORANDI ${ }^{3}$, STEFANO TAMASCELLI ${ }^{4} \&$ BENJAMIN COHEN $^{5}$ \\ ${ }^{1}$ ICCS, Athens, Greece \\ ${ }^{2}$ IHE, Delft, The Netherlands \\ ${ }^{3}$ U-Hopper, Trento, Italy \\ ${ }^{4}$ Xteam, Rovigo, Italy \\ ${ }^{5} \mathrm{IBM}$, Haifa, Israel
}

\begin{abstract}
Flood risk prediction has been traditionally based on models that are developed from time-series of data collected over long periods of time from expensive and hard to maintain in-situ sensors available only in specific areas. The climate change has made the monitoring of the flood events imperative and has raised the question of whether the development of flood models can be disengaged from the in-situ sensors. The Scent toolbox is based on smart collaborative and innovating technologies that augment costly in-situ infrastructure, enabling citizens to become the "eyes" of the policy makers by monitoring Land Cover/ Land Use (LC/LU) changes in their everyday activities and environmental phenomena like floods by crowdsourcing relevant information. Experts in the field of flood models define areas of interest through a specifically designed tool and ask volunteers to collect specific data needed at these areas using engaging gaming applications. These data may include images that are processed through an Intelligent engine and classified based on a LC/LU taxonomy, video of floating objects and images of water level indicators that allow the automated extraction of the water velocity and the water level and sensor measurements with low-cost portable environmental sensors. It will be described in detail how the volunteers are engaged to collect these data, how the data are validated, and how they are used to create improved LC/LU maps and contribute to the development of improved flood models reducing the cost and infrastructure needed.
\end{abstract}

Keywords: scent toolbox, crowdsourcing observations, OGC-compliant data handling.

\section{INTRODUCTION}

Europe has invested a lot in infrastructure to achieve an accurate Earth observation capacity. Initiatives such as Copernicus provide a mapping of forest areas, wetlands or artificial surfaces; yet, the burden of investing in new equipment or maintaining the current infrastructure is unsustainable. Ways of complementing the in-situ infrastructure with citizen-sourced data at a low cost are currently investigated. Recognising that citizen participation in environmental policy making is in its infancy and that citizens feel unable to influence environmental policies the Scent toolbox aims to alleviate this barrier. Through a constellation of smart technologies, it enables citizens to support the policy makers by monitoring LC/LU changes as part of their everyday activities augmenting the in-situ infrastructure with a people-generated observation web.

In order to achieve its goals, the Scent toolbox is based on an innovative architectural design that is presented in Fig. 1, where key components dedicated to specific tasks are connected in a way that allows the flow of information from local authorities, to volunteers, to environmental experts and back to the authorities that can now have improved monitoring of the phenomena of interest allowing them to make educated decisions that can help and support the areas. The key components of Fig. 1 are briefly presented here. 
A crowd-sourcing platform that provides a series of tools and applications that allow the flow of information between the components of the toolbox as well as the creation of information from policy makers and contributions from the volunteers. It includes a dedicated tool (Authoring tool) for policy makers that allows them to identify areas of interest, create campaigns and Points of Interest (PoIs) and access the collected and extracted information in a user-friendly way as map overlays. Part of the crowdsourcing platform is also a series of gaming applications that aim to engage volunteers to collect images and sensor measurements as defined in the Authoring tool and to contribute to environmental monitoring by providing, qualifying and interpreting information about LC/LU.

An intelligence engine, which uses innovative machine learning techniques to classify and annotate images from citizens and open platforms. The classification is done with respect to the Scent taxonomy, which is based on the CORINE taxonomy but is also enriched to include elements needed for the flood models. To fully utilize the collected and extracted information, the validated data are used to create improved LC/LU maps. In addition, in order to support the collection of river measurements in a uniform way, useful to the flood models and invariant of the experience of the volunteers to collect scientific data two tools have been developed. The tools use state of the art image recognition algorithms that extract from multimedia (video and image) water level and water surface velocity measurements.

A collection of environmental sensors that are going to support the collection of the data needed for the flood models. The sensors will range from in-situ sensors that are available in the areas of interest, portable sensors that the volunteers use to collect additional measurements as well as sensors mounted on drones flying over the areas of interest.

A Harmonisation platform that collects all the crowdsourced observations, transforms them to OGC compliant observations, stores them with respect to time and space as such and offers them to the Scent toolbox components as needed as well as to GEOSS as web services making the collected data findable and re-usable. Part of the Harmonisation platform is also the Data Quality Module that assigns a trust level to every registered user and sensor.

The Scent toolbox will be evaluated for a year during a series of campaigns starting the summer of 2018 in two large scale pilots of great environmental impact; the urban site of Attica Kifisos River and the rural site of Danube Delta. The two pilot areas have been chosen carefully for their specific characteristics as well as their different needs and topology.

Finally, the collected data along with the extracted information from the different components will be used to improve the flood models of the areas, quantify the impact of LC/LU changes to flood maps and spatio-temporal flooding patterns enabling more effective flood-related planning and management by policy makers.

\section{CROWDSOURCING PLATFORM}

The Crowdsourcing platform includes four main modules of the Scent Toolbox:

- The Crowdsourcing backend, which handles communication and interaction of crowdsourced content among the platform components.

- The Authoring tool, which represents the entry point for local authorities to (i) define and customise citizen engagement campaigns on LC/LU data collection (ii) access crowdsourced images and citizen notifications (iii) view and explore the extracted information from the crowdsourced data.

- The Open Image tool, which crawls open image repository for relevant content, in order to augment the crowdsourced data with already available information.

- The gamification applications that aim to engage citizens into contributing data. 


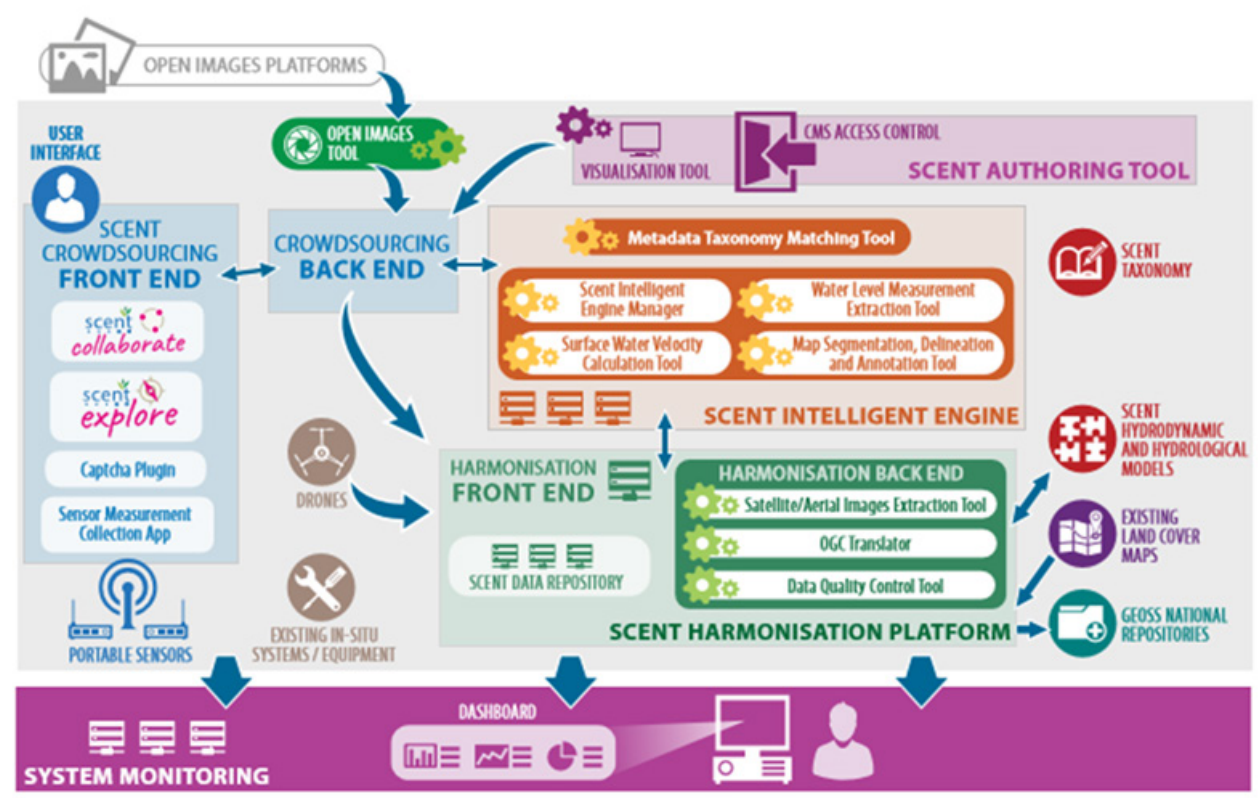

Figure 1: Architectural overview of Scent toolbox.

\subsection{Crowdsourcing backend}

The Crowdsourcing backend caters for the upload and management of citizen-generated data such as images, videos, annotations and questionnaires. It supports the flow of information [1] from the policy makers (Authoring tool) to the volunteers (gaming applications), to the Intelligent engine and to the Harmonisation platform. In order to ensure that the produced data are of high quality a functionality that validates the annotations (given either from the volunteers or the Intelligent engine) is implemented allowing the data that are sent to the Harmonisation platform for storage to be considered final. The implementation supports the following set of functionalities:

- Data quality management: the quality of the classifications for each image is checked and it is decided whether further annotations (from the dedicated gaming applications) are required in order to meet the overall Scent quality assurance standards, or whether the classifications provided can be considered finalised.

- Provide images to annotate to the various frontends: when the available annotations are not satisfactory and should be complemented by additional ones the content (and metadata) become available for annotation to the gaming applications.

- Collects (annotated) images/videos from various sources: all user-generated content is sent to the Crowdsourcing backend, which handles and manages it, making sure it is forwarded and/or made available to the relevant Scent toolbox components.

- Provide campaign configuration data to the gaming applications (received from the Authoring tool): campaigns are configured by policy makers at the Authoring tool, stored at the Crowdsourcing backend and forwarded to the gaming applications. 
- Provide raw crowdsourced data to the Harmonisation platform: crowdsourced data uploaded to the Crowdsourcing backend are made available to the Harmonisation platform through a purposeful interface.

\subsection{Open Image tool}

The Open Image tool provides crawling functionality by querying a predefined set of open image platforms when policy makers decide to use images provided by third parties under an open data license in order to complement Scent-generated data. Configuration data (bounding box for position and time range for timestamp) are provided by the policy makers through the Authoring tool. The Open Image tool provides the functionality of querying open image platforms for relevant images based on the configuration data.

\subsection{Authoring tool}

The Authoring tool provides a user-friendly web interface, as shown in Fig. 2, for policy makers, enabling them to create, view, edit or delete content related to Scent Campaigns as well as PoIs. It is responsible for managing the policy makers' user accounts, their personal settings as well as for notifying them of any relevant reported events. It also keeps the users informed of important events (account changes) through logs. In addition, it gives to the policy makers access to crowdsourced images and sensor measurements as well as maps of the areas of interest with information regarding LC/LU, flood models, Campaigns and/or questionnaires. The Authoring tool implementation therefore supports the following set of functionalities:

- Manage user accounts: The users of the Authoring tool are separated in two categories, policy makers and premium users, with different privileges.

o The policy makers can create and view Campaigns and PoIs, they can also edit and delete any Campaign and PoI that is in the system. They can add/delete a premium user and give administrative privileges to a premium user. The policy makers can also create a questionnaire and reviewed the collected information in a structured way.

o The premium users can create and view Campaigns and PoIs but they can only edit Campaigns and PoIs that they have created.

- No unregistered user is allowed access to any of the available information.

- Manage personal settings: The Authoring tool allows the users to save their personal settings such as language and place of interest for their accounts.

- Manage Campaigns and PoIs: The Authoring tool gives the users the ability to handle their own Campaigns through their accounts. In addition, provides users the ability to create, view, edit or delete both PoIs and Campaigns using the map of the area of interest. Users can also choose to use a PoI in order to provide critical or important information for the area rather than request information.

- Access user-generated data and sensor information: The users can access crowdsourced images, sensor measurements and taxonomy metadata through a purposeful web interface.

- Visualise flood risk maps and LC/LU maps: The users can have an overview of a place of interest through a map. The Authoring tool shows (as layers over the original map) the flood risk map and/or the LC/LU map. 
- Inform users of questionnaire results: The users can see the information collected as answers to the questionnaires that have been set by any user in a structured way.

- Initiate the Open Image tool: The users may require an updated LC/LU map for an area of interest. Through the Authoring tool the bounding box of the area and the time of interest are defined. The configuration data are sent to the Crowdsourcing backend to be handled accordingly.

\subsection{Gamification apps}

A series of data and actions have been defined as very important for the monitoring of the $\mathrm{LC} / \mathrm{LU}$ changes and the improvement of the flood models, these data have been mapped to straightforward, time-effective and meaningful actions that the volunteers can carry out to collect them [2], [3]. These are actions can be described as follows. (i) Annotate, with tags from the Scent taxonomy, images collected by the Open Image tool. (ii) Take an image of specific objects in a predefined area (e.g., vegetation at the river bank, waste and brought materials in the manholes, tree banks/branches, dustbins, cars and vehicles along the river bank or in smaller streams connected to the main river). (iii) Take an image or video in a very specific point of interest (e.g, image of a water level indicator, video of a predefined floating object). (iv) Answer to questionnaires at areas of interest to support the collection of structured data. (v) Use portable sensors to take some environmental measurements.

Based on the above identified actions four independent applications have been designed to simplify the process, boost the user engagement and collect properly formatted data of high quality.

\subsubsection{Explore app}

Scent Explore is a mobile application that the volunteers use to locate specific areas of interest, find each point where specific tasks should be carried out, and gain rewards when successfully concluding them. Two reward mechanisms (points and badges [4]) are used to boost the user engagement. The tasks that the users are asked to carry out and for which they are rewarded are: (i) Take a picture containing a specific object from the Scent taxonomy; (ii) Go to a specific location and finding the "Little Animals".

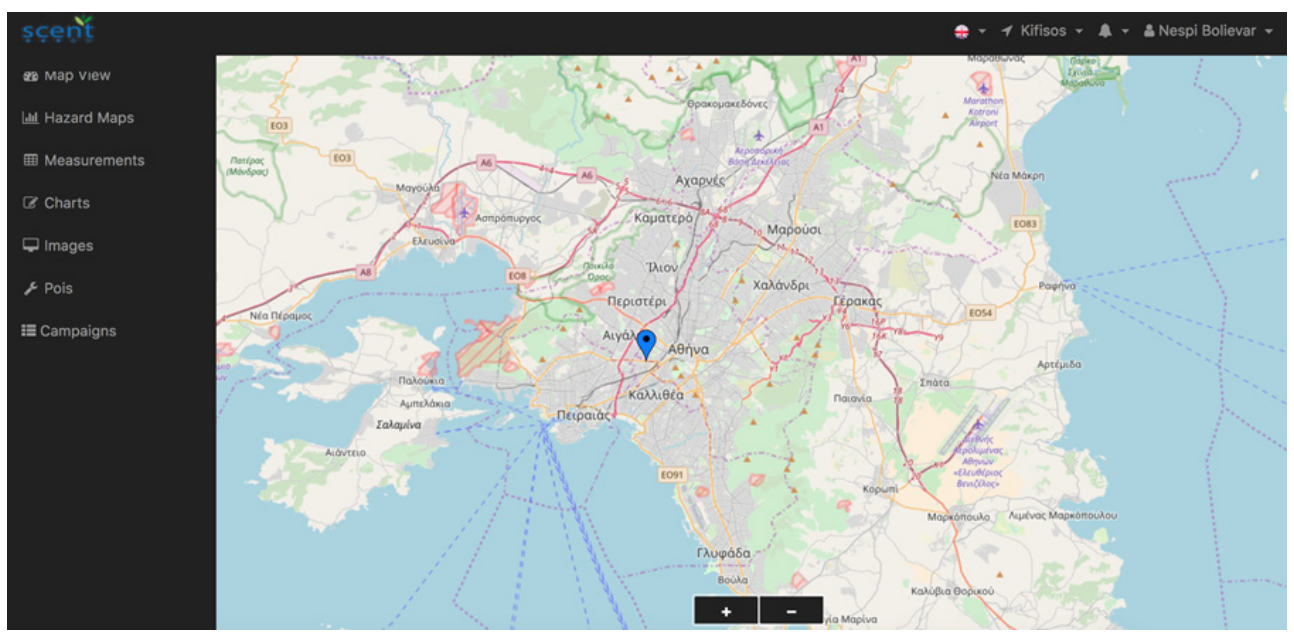

Figure 2: Scent Authoring tool. 
This functionality will allow the user to spot the exact place where a water level indicator is located, or where a sensor measurement should be carried out. (iii) Answer questionnaires regarding the area.

The user can either play as guest or as registered user; while it has been decided to have a single database for user profiles. This means that the users who register through the app, can also use the same account for the other gaming applications having a consistent user experience. Another functionality of the Explore app is that when a user is near a "little animal"/PoI, even if she is not looking at the map and/or the phone is in standby mode, the device will vibrate, the camera will automatically turn on and the user will be invited to take a picture of the area. The user will then be asked to tag the picture with elements from the Scent taxonomy. The user can also take and tag pictures of areas and objects which do not correspond to predetermined points of interest; allowing the user to contribute at any given moment data that she feels are of interest. When the user looks into the camera while searching for a little animal, the GPS position will be integrated with the vector data of the gyroscope or with data coming from the accelerometer guiding the user to locate the 'little animal' and as a result take images with specific orientation that will include information of interest. The user interface of the application is presented in Fig. 3.

\subsubsection{Collaborate}

The Scent Collaborate is a browser-based crowdsourcing platform that allows users to annotate existing pictures choosing elements from the Scent taxonomy that exist in them. The same picture can be annotated by more than one user so as to support the validation of the annotations from the Crowdsourcing backend. Here too the same two reward mechanisms are applied as the user is rewarded with points based on the number of annotated pictures. Once more the points are connected to badges that acknowledge the achievements of the users. Scent Collaborate is oriented toward a broad audience interested in joining the Scent movement even if they are not living close to an area of interest. The platform targets citizens interested in environmental issues, contributing to science and supporting the policy makers.

\subsubsection{Captcha}

This is a browser-based plugin to be integrated in third-party websites when there is a need to verify that the user is actually a human and not a robot. The user is asked to spot which of the images presented to her include a tag from the Scent taxonomy, the response is used to support the validation mechanism for the annotations.

\subsubsection{Sensor measurement collection app}

This is a mobile application that is used in addition to the Explore App to communicate with the portable sensors available at the area of interest, record the measurements and send them to the backend. The users can login using their available account and collect rewards for their contributions. The same two reward mechanisms are applied here as well with the user getting points based on the number of sensor measurements. Once more the points are connected to badges that celebrate the achievements of the users.

\section{INTELLIGENT ENGINE}

The Scent Intelligent engine uses state of the art machine learning techniques in order to ensure that all the available information included in the multimedia collected by the volunteers is fully utilised. 


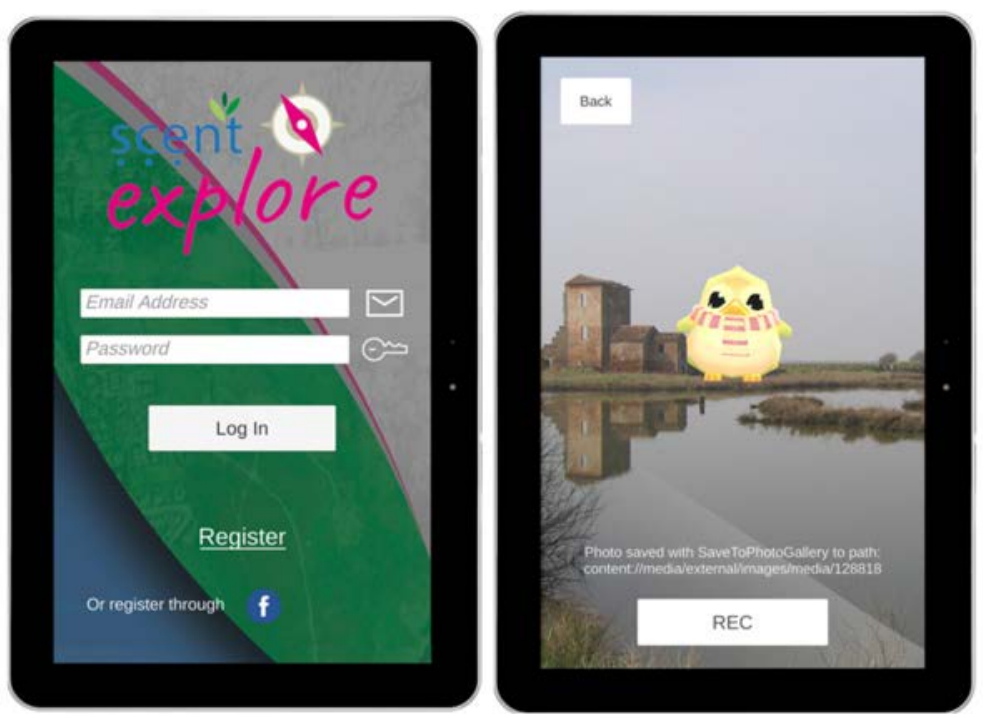

Figure 3: Scent Explore app.

\subsection{Image Analysis tool}

The Image Analysis tool (IAT) will analyse the input image and classify items seen in the image as belonging to the elements of the Scent taxonomy. The classification process will be handled by a specifically developed Deep Learning (DL) network optimized for Scent oriented taxonomy classifications. The DL network will be trained with representative images collected from open image platforms and manually annotated with respect to the Scent taxonomy. Use of other existing broad-category image classification tools (e.g. IBM Watson Visual Recognition service) will be investigated as a means for improving the accuracy and confidence level of the classification. In addition, the IAT will receive the information collected from the Crowdsourcing backend validation mechanism for incremental learning purposes.

\subsection{Water level and water velocity measurements from multimedia}

In order to get the river measurements with a consistent and accurate way from volunteers that have no technical knowledge two tools have been designed.

The first one is the Water Level Measurement tool which uses state of the art image recognition techniques in order to extract the water level from images containing a water level indicator that is half-submerged into water. The initial goal of the tool is to "read" the indicator and extract the number that is closer to the water lever. In order to achieve that the tool extracts features of the input images and match them with pre-calculated model's invariant of rotation, luminance variation, image noise or scale that are stored in a model database. If the quality of the image does not allow for the recognition of the numbers of the indicator, calibration techniques based on the patent of the indicator and tis bounding box are exploit resulting in a less trusted estimation of the water level.

The Water Velocity Calculation tool uses state of the art video processing algorithms in order to extract the water surface velocity from a video containing a pre-defined floating 
object moving on the surface of a water body [5]. The Kalman filter is used for the object localisation, while the object displacement is estimated based on feature matching algorithms and calibrated using the known dimensions of the floating object. The tool is resistant to noise introduced to the video by intentional or unintentional movement of the camera as long as the floating object is included in the video frames. The more stable elements from the surrounding environment (such as trees and rocks) the video includes the more accurate calculation of the estimation of the displacement can be achieved.

Each measurement estimation extracted by the tools is accompanied by a degree of trust that highly depends on the quality of the input data. The tools have been designed so that a high degree of trust can be achieved from images and videos taken from regular smartphones.

\subsection{LC/LU map overlays}

There are many approaches and algorithmic tools that are adequate for effectively performing map segmentation regarding $\mathrm{LC} / \mathrm{LU}$; their applicability however is closely related to the classification problem and the characteristics of the study area. After a study of the available aerial images of the areas of interest and preliminary data exploration, it has been concluded that the preferable way of performing the map segmentation is to handle it as an aerial scene classification task. Accordingly, the map under consideration is split into overlapped tiles of predefined size, which are slid across both vertical and horizontal directions, aiming to build one or more classifiers that are capable of classifying all the tiles according to the Scent taxonomy.

\section{ENVIRONMENTAL SENSORS}

Environmental sensors aim to collect the detailed set of environmental and operational parameters that are important to both policy makers as well as hydrology experts. Important measurements collected for further use and exploitation include soil moisture, air and water temperature. In this context innovative sensors are deployed and adapted as part of the Scent toolbox. Furthermore, environmental sensors and digital cameras are integrated on multicopters based on open source hardware platforms for obtaining spatially continuous data, including elevation data for quantitative mapping and monitoring parameters with an increased accuracy.

In-situ sensors: The majority of environmental data is traditionally collected through insitu monitoring stations. Not wanting to exclude the information that might be available from such stations in areas of interest water level and water velocity monitor station that are available to the pilot areas are integrated with the Harmonisation platform providing measurements which are stored as OGC compliant observations. These measurements when available serve two very important roles. On the one had they provide the ground truth for the Data Quality Control module allowing for better and with higher degree of trust definition of the thresholds used to judge a measurement. On the other hand they provide continuous data that can be incorporated to the flood models using traditional techniques.

Portable sensors: An off-the-shelf sensor has been chosen as the portable sensor that the volunteer will use for the sensor measurements. A list with some very important and critical for the user engagement criteria was composed to assist on this choice. The criteria included restrictions regarding portability, reliability, ease in use, low-cost and connectivity. The sensors are currently available and integrated with the Measurement Collection App ready to be used in the first pilot activities.

Drone sensors: Ready-to-fly digital camera and environmental sensors mounted on a multicopter will be deployed during the pilot activities for a quantitative mapping of 
environmental parameters that will cover air, land and water parameters. The multicopter will take photos of the regions in orthomosaic and DEM files as well as in near-IR. Parameters will be collected as digital aerial photography that spatially complements ground-based data. The UAV will measure also temperature by using a thermal camera. Images in near-IR and orthomosaic will be used in the improvement of the flood models. In order to improve the location accuracy a differential GPS will be used.

\section{HARMONISATION PLATFORM}

The harmonisation platform is a scalable web-based platform that stores IoT as OGC compliant observations and offers them as a web service while at the same time verifying the quality of the data and extracting a trust level for the sensors and users involved.

\subsection{OGC compliance}

The choice for storing the data as OGC observations was made based on the importance of making the collected information findable and reusable. Based on that the Harmonisation platform has been designed to receive as input IoT-data, which are sensor measurements, custom metadata from images and videos, drone-generated images/measurements and map overlays. The platform translates the aforementioned data and metadata to OGC compliant observations using the applicable standard, such as SOS, SES, WMS, WFS and SensorML. The OGC compliant observations are then stored in a distributed and secure environment, indexed with respect to time and space and are accessible using CQL (Common Query Language) always with respect to the demands of the OGC standard [6].

\subsection{Data Quality Control}

The Data Quality Control system is aiming to cope with the following main challenges: correctness/accuracy of the sensor measurements, user reliability and protection of the system from malicious contributions. In order to handle the volume of the data, a filtering process flags as invalid a percentage of the input data that do not fall within the expected range. During this step, it is preferable to flag a valid result than allowing an invalid one to enter the system. Depending on the value measured, the area, the availability of historical measurements and the volume of the data, different solutions can be applied to deal with this issue. In the context of Scent, two basic approaches are implemented:

- Gaussian probability distribution: Provides data plausibility tests (range test, sigma test, delta test) employing different statistical methods (standard deviation, variance, etc.). For these tests, the calculation of a threshold parameter poses the most critical challenge, being determined by statistical distributions based on existing data over a period sufficiently long to capture the full suite of variability [7], [8].

- Fuzzy logic: Enables the possibility to encode linguistic rules and heuristics, reducing the solution time since the expert's knowledge can be built in directly. In addition, its qualitative representation form makes fuzzy interpretations of data natural and an intuitively plausible way to formulate and solve problems [9], [10].

The next step is to scrutinize the flagged results to see if they correspond to invalid or marginal data. Extra logic rules are implemented to distinguish the two cases. These rules try to connect the available information based simply questions such as: Is this the only measurement from the area/time flagged? Was this measurement from a trusted user/ reliable sensor? Was the sensor calibrated/maintained recently? 
User-wise, an ELO rating scheme is implemented, allowing the system to judge the users regarding the quality of the data that they produce. By keeping an internal score of trust towards each user, the system can be protected from harmful intents, whilst keeping the data without noise. For instance, wins against the same ELO rating all the time will result in the user's rating reaching a maximum value and become invariable. Moreover, the ratings for rewards/ punishments are chosen in a way that the novice user will be rewarded a lot when he does something right and lose a little when he does something wrong. This adapts as the expertise level increases protecting the system from malice while not punishing inexperience. Last but not least, detecting malice contributions mixed within valid ones can be achieved by a periodic check about ELO ratings that are stable while the user contributes a lot as this means that the user is introducing a lot of noise to the system.

\section{PILOT ACTIVITIES}

The two regions where the Scent toolbox is going to be test are the Danube Delta in Romania and the Kifisos River basin in Athens, Greece. The two pilot areas were chosen carefully in order to test the toolbox in two fundamental different topologies of areas with different needs and challenges. In the Danube Delta in Romania, the flooding is a necessary and welcome part of maintaining the ecosystem, as the unique ecosystem of the area with the vibrant wildlife and the remarkable plant life, is sustained by these waters. There it is important to understand how these flood waters work, how the climate changes and the changes in the $\mathrm{LC} / \mathrm{LU}$ of the area affect the phenomena and what measures should be taken for the sustainability of the ecosystem before it is too late.

In the Kifisos River the challenges are completely different given the urban environment. The landscape is changing fast and dramatically as streams are covered with concrete, forests are torn down and the natural course of the river is modified. There it is very important to understand the river, its course and the LC/LU changes. Measures should be taken to avoid any overflows of the river that affect the communities living close by.

Preparatory activities for the execution of the two pilot demonstrations have already been initiated. From August 2018 onwards, several thematic campaigns, focused on the collection of LC/LU images, sensor measurements and river data, will take place in each pilot area, where volunteers will be engaged with specific test cases and tools. The pilot activities will run for a period of approximately 10 months, foreseeing at least 6 different campaigns to take place in each pilot area. The concept of the thematic campaigns was carefully chosen, to allow the organization of a dedicated workshop at the start of each campaign where volunteers will not only be informed about the project and but also be trained in the use of the Scent toolbox used during the campaign.

\section{FLOOD MODELS}

When floods are the problem to be addressed, models can serve as a base for discussion and evaluation of possible adaptive measures to control their impact on the environment. In this context, the Scent toolbox includes models representing hydrology and/or hydrodynamics of flow, in relation to flood events, such that data used for their simulation will be extended with contributions from citizens (crowdsourced data). Data collected by citizens will be integrated into the models and tested for its validity.

Initially, flow models of two selected pilot study areas are built with existing data, acquired without any contributions from citizens. These two models are compared, with the models enriched with data collected by citizens. The two pilot case studies under consideration have different characteristics, hence they require different modelling approaches. The tools used for model building are part of a non-commercial modelling suite 
developed by the Hydrologic Engineering Centre (HEC) of United States Army Corps of Engineers (USACE); the river analysis system (HEC-RAS) and the hydrological modelling system (HEC-HMS). These two software packages are freely available for download and were chosen based on their capabilities to represent complex river network systems (such as Danube Delta) and fast occurring floods (such as Kifisos catchment).

The emphasis in the Danube Delta [11] pilot is on determining the spatio-temporal flooding patterns which are important for the ecosystem of the delta. This requires a detailed 1D/2D hydrodynamic model, which represents the flow of water through channels, lakes and over the land. For the whole area of the Danube Delta, the set of built models based on combinations of different geometry and/or boundary and initial conditions. Three types of hydrological regimes are taken into consideration: dry, average and wet year, with corresponding flows on the main Danube delta branches.

In Kifisos pilot [12] the emphasis is related to flood as a hazard. Based on flood behavior in this area, combined with data availability a hydrological model that can generate flood runoff is considered for the upstream part of the catchment. A smaller hydrodynamic model is also considered for the last $3 \mathrm{~km}$ of the catchment. The hydrological and hydrodynamic models were developed and tested separately, however the input for the upstream boundary condition of the hydrodynamic model is received from the hydrological model. The connection of the two developed types of models is shown in Fig. 4.

The developed models have been developed and tested with existing data. However, they still have significant uncertainties that will be addressed with the crowd-sourcing campaigns of Scent. In Danube Delta, the additional LC/LU data will be used to provide improved roughness parameter, and other data will validate the model in terms of spatial distribution of flood extent, flood levels and flood velocities and discharges, calculated by the 1D-2D HEC-RAS model. In Kifisos the LC/LU data contributed by citizens will be used for updating infiltration and runoff parameters of the rainfall runoff model built by HEC-HMS and improved cross section data and updated measurements of water levels and velocities/discharges in the river will be used to better calibrate and validate the HEC-RAS hydraulic model of the downstream part of the catchment.

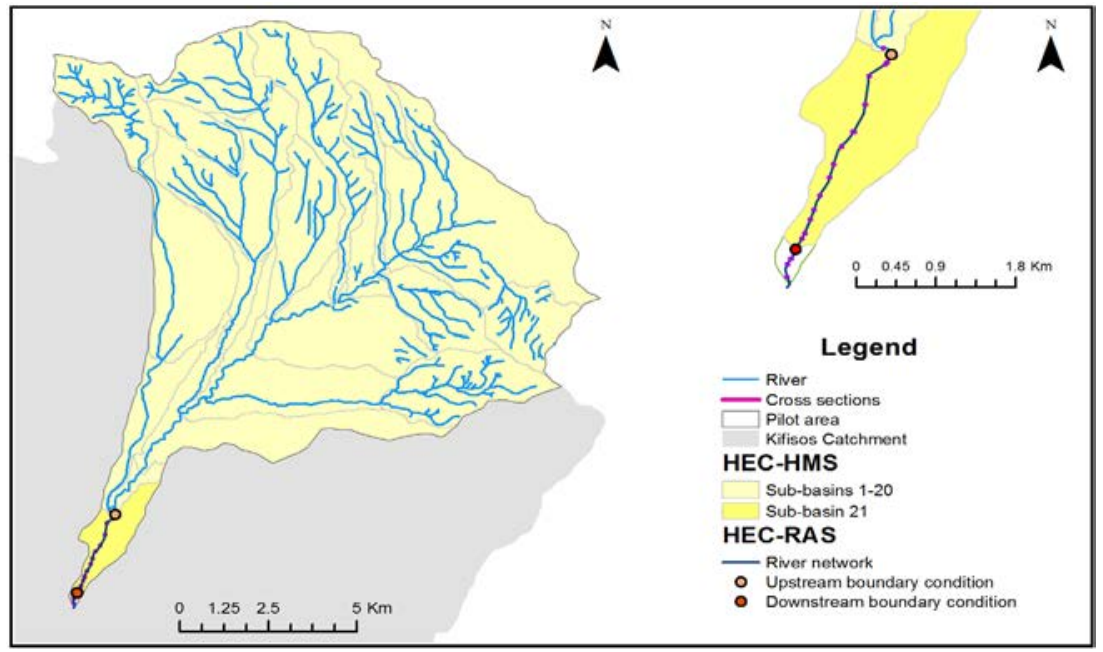

Figure 4: Connection of HEC-HMS and HEC-RAS model. 


\section{CONCLUSIONS}

It has been presented above that careful planning along with an innovative architectural design and a constellation of smart technologies can contribute to better decision-making through the empowerment and active role of citizens in environmental monitoring. The Scent toolbox is allowing citizens to contribute the proper scientific data as needed for the researchers, resulting in the production of improved flood models. These models become invaluable tools for the policy makers in order to make educated decisions regarding local preparations for the wet season.

\section{ACKNOWLEDGEMENT}

This paper is supported by European Union's Horizon 2020 research and innovation programme under grant agreement no 688930, project SCENT (Smart Toolbox for Engaging Citizens into a People-Centric Observation Web).

\section{REFERENCES}

[1] Carreras, I. et al., Crowd-sensing: Why context matters. Pervasive Computing and Communications Workshops (PERCOM Workshops), 2013 IEEE International Conference on IEEE, 2013.

[2] Fucks, M., Fizek, S., Ruffino P. \& Schrape N., Rethinking Gamification, Meson Press, 2014.

[3] Dörner, R., Göbel, S., Effelsberg, W. \& Wiemeyer, J., Serious Games: Foundations, Concepts and Practice, Springer, 2016.

[4] Chou, Y.-K., Actionable Gamification - Beyond Points, Badges, and Leader Boards, Octalysis Media, 2015.

[5] SelvaBalan, M., Sharma, N., Deshpande, G., Kankariya, C. \& Naik, A.A., Surface water velocity measurement using video processing: A survey. 2014 International Conference on Electronics and Communication Systems (ICECS), Coimbatore, 2014.

[6] Open Geospatial Consortium (OGC), Online. www.opengeospatial.org/.

[7] Taylor, J.R. \& Loescher, H.L., Automated quality control methods for sensor data: A novel observatory approach. Biogeosciences, 10, pp. 4957-4971, 2013.

[8] Fiebrich, C.A., Morgan, C.R., McCombs, A.G., Hall, P.K. Jr., \& McPherson, R.A., Quality assurance procedures for mesoscale meteorological data. J. Atmos. Oceanic Technol., 27, pp. 1565-1582, 2010.

[9] Mahajan, A., Wang, K. \& Ray, P.K., Multisensor integration and fusion model that uses a fuzzy inference system. IEEE/ASME Trans. Mechatron., 6(2), pp. 188-196, 2001.

[10] Timms, G.P., de Souza, P.A., Reznik, L. \& Smith, D.V., Automated data quality assessment of marine sensors. Sensors, 11(10), pp. 9589-9602, 2011.

[11] Giosan, L., Constantinescu, S., Filip, F. \& Deng, B., Maintenance of large deltas through channelization: Nature vs. humans in the Danube delta. Anthropocene, 1, 3545. DOI: 10.1016/j.ancene.2013.09.001, 2013.

[12] SelvaBalan, M., Sharma, N., Deshpande, G., Kankariya, C. \& Naik, A.A., Surface water velocity measurement using video processing: A survey. International Conference on Electronics and Communication Systems (ICECS), Coimbatore, 2014. 